\title{
Triple-negative breast cancer: one or more entities?
}

\author{
Alexios Matikas ${ }^{\# * 1}$, Georgios Lazaridis ${ }^{\# 2}$, Sofia Agelaki \\ ${ }^{1}$ Department of Medical Oncology, \\ University Hospital of Heraklion, Crete, Greece \\ 2University Medical Oncology Ward, Aristotle University of Thessaloniki, \\ Papageorgiou General Hospital of Thessaloniki, Greece.
}

\begin{abstract}
Characterized by an aggressive clinical course and relatively poor prognosis, triple-negative breast cancer (TNBC) refers to a diverse group of tumors with distinct molecular characteristics rather than to a single entity. The recognition of distinct gene expression subtypes within the group of TNBC and the description of an ever-expanding set of genetic events has led to improved understanding of the underlying biology. However, the improvement of clinical results has been incremental despite undergoing efforts to evaluate the role of molecularly targeted agents in the treatment of TNBC. The relative rarity of each one of these genetic events increases the difficulty of conducting large clinical trials, further hindering our ability to identify meaningful, personalized treatment approaches. Herein, we summarize current knowledge on TNBC, focusing on molecular pathology and emerging treatment approaches.
\end{abstract}

Keywords: Breast cancer • Triple negative $\bullet$ Basal-like $\bullet$ Subtype $\bullet$ Gene expression

(c) De Gruyter Open

\section{Introduction}

Breast cancer is the most frequently diagnosed cancer in the world and the most common cause of cancer-related death among women [1]. Breast cancer was considered a single homogenous entity for almost a century, until advances in the understanding of the underlying biology during the last 25 years revealed the great heterogeneity of the disease. Moreover, treatment advances such as newer chemotherapy regimens and tailored treatment according to hormone receptor and human epidermal growth factor receptor 2 (HER2) status led to improvements in disease specific and overall survival. Despite this considerable progress, a subgroup of breast cancers, categorized as "triple negative", currently lack recognized targets for molecular-oriented therapies.

Triple-negative breast cancer (TNBC) is defined as a primary breast adenocarcinoma, which lacks expression for estrogen receptor (ER) and progesterone receptor by immunohistochemistry (IHC) and HER2 overexpression or amplification by $\mathrm{IHC}$ or in situ hybridization, respectively $[2,3]$. First recognized as a distinct breast cancer subgroup in 2005 [4], TNBC accounts for approximately $10-20 \%$ of newly diagnosed breast cancers. TNBC has a distinct epidemiology, histology and clinical course, whereas there is an increasing recognition of its significant heterogeneity. In general, TNBC is associated with a particularly poor prognosis despite its relative chemosensitivity, a fact partly attributed to its unique biological characteristics and to the lack of approved targeted treatments for this breast cancer subtype [5]. However, the delineation of the pathways that drive TNBC has led to the development of novel treatment strategies that have shown promise in clinical trials and it is hoped that these may prove beneficial to patients with TNBC.

In this review, we summarize the current state of knowledge of TNBC, including its epidemiology, natural history, pathology, molecular biology and treatment and provide an update on novel therapeutic approaches. 


\section{Epidemiology}

Several risk factors that are associated with the development of TNBC have been described during the previous years and these include [6]:

Age: Women under the age of 40 years old have a twofold increased risk for TNBC (Odds Ratio OR 2.13, 95\% Cl 1.34-3.39) [7]. The effect of age was also shown in another study where patients with TNBC tended to be of younger age compared with patients with luminal $A$ breast cancer $(P<0.0001)$ [8].

Race: The effect of race in the risk for TNBC has been well described in several studies. In a report by Stead et al, the odds of having TNBC were threefold higher in African Americans compared to white women, regardless of menopausal status and obesity [9]. Also, in an analysis of 496 cases from the Carolina Breast Cancer Study, the researchers concluded that premenopausal African American women had the highest prevalence of TNBC followed by postmenopausal African American women and premenopausal white women [10], a finding that may explain the poorer prognosis of breast cancer among this ethnic group. Interestingly, among 507 women divided in two cohorts in Nigeria and Senegal, only $25 \%$ of cancers were ER positive; the most common molecular subtypes were basal like (27\%) and unclassified triple negative (28\%), compared to $28 \%$ that were luminal $A$ or $B$ [11]. The underlying mechanisms of the increased risk for TNBC among women of African descent are unclear.

Hormonal related factors: Premenopausal status has been found to correlate with increased risk for basal-like breast cancer, a molecular subtype that significantly overlaps with TNBC [10,12]. However, the age of menarche and menopause have not been correlated to the risk for TNBC in contrast to hormonedriven breast cancers. Moreover, contrary to previously described risk factors for ER-positive breast cancer, nulliparity seems to have a protective effect for TNBC (Hazard Ratio $=0.61,95 \% \mathrm{Cl} 0.37-0.97)$, whereas the risk increases in association with the number of births [13]. The examination of the effect of breastfeeding on the risk for TNBC yields conflicting results, with one study showing a protective effect and no effect in another [12,13]. Moreover, the use of contraceptives was associated with a significant increase of TNBC among women under 40 years old in one study [14], but not in another [13].

Obesity: The association between obesity and TNBC has been described in several studies $[7,10]$ and was confirmed in a meta-analysis showing that a body mass index over 30 correlated with increased risk for TNBC
(OR 1.2, 95\% Cl 1.03-1.40). The effect of obesity in this meta-analysis was found to apply to premenopausal women only (OR 1.43, 95\% Cl 1.23-1.65) [15]. On the other hand, at least one study did not find an effect of obesity on risk for TNBC [9].

Smoking and alcohol: Several studies support the role of alcohol consumption and smoking as risk factors for breast cancer [16-19]. However, a study of women enrolled in the Women's Health Initiative confirmed their role as risk factors for ER-positive breast cancer but found that smoking did not alter TNBC risk and alcohol had a protective effect [20].

\section{Pathology and natural history}

The most common histology of TNBC is infiltrating ductal carcinoma, not otherwise specified; medullary carcinoma is also more commonly triple negative [21]. Moreover, rare histologic types, such as adenoid cystic and secretory carcinomas that follow a more indolent course and the aggressive metaplastic carcinomas, are commonly triple negative [22]. Triple-negative tumors tend to be larger on presentation [23], are usually of high grade, present significant atypia and exhibit specific morphologic features, such as increased mitotic counts, geographic necrosis, pushing border of invasion and stromal lymphocytic response [21]. In contrast to other breast cancer subtypes, even small triple-negative tumors can be node positive ( $55 \%$ of women with $<1 \mathrm{~cm}$ TNBC in one study) [23], whereas nodal positivity does not increase with size in the same manner as in ERpositive tumors.

TNBC is rarely diagnosed mammographically, which may be due to its detection in younger women and its aggressive growth [23]. Moreover, ER-negative tumors more frequently present as interval breast cancers relatively to ER positive ones. [24]. Like any other breast cancer subtype, the staging of triple-negative tumors follows the American Joint Committee on Cancer - International Union for Cancer Control (AJCC-UICC) tumor node metastases system, currently in its seventh version [25].

Overall, triple-negative tumors carry a poor prognosis compared to other breast cancer subtypes [23,26,27]. In a study of 15,204 women with breast cancer, 2569 women with TNBC were identified. Overall survival (HR 2.72, 95\% Cl 2.39-3.10) and breast cancer-specific survival (HR 2.99, 95\% Cl 2.59-3.45) were significantly shorter for patients with TNBC, a difference that was most pronounced during the first 2 years after diagnosis (HR 6.10, 95\% Cl 4.81-7.74). Also, patients with TNBC 
are more likely to develop visceral (especially lung) and brain metastases but less likely to develop bone metastases compared to ER-positive tumors [27,28]. Moreover, the survival curves of TNBC patients differ greatly compared to those of ER-positive breast cancer patients: there is a dramatic increase of the risk of death during the first 3-5 years after diagnosis but recurrences after 10 years are rare [26,28], implying that some patients may be cured. Interestingly, in a retrospective single-center study, Hamm et al found that when adjusted for age, stage, grade, size of tumor and treatment, women with TNBC had the same prognosis as non-TNBC ones [29]. This study and others [30] underscore the fact that size is an important predictor of survival for TNBC.

\section{Molecular subtypes of triple-negative breast cancer}

In their seminal work, using cDNA microarray analysis, Perou et al divided breast cancer in distinct molecular intrinsic subtypes. Luminal A and luminal B express genes found in luminal cells and widely overlap with ER-positive cancers, HER2 enriched that accounts for most HER2 positive cancers, normal breast-like (not widely adopted since it could be due to contamination of samples with normal tissue) and basal-like subtypes, which present a significant overlap with TNBC [31]. Pathology-based surrogate definitions of these intrinsic subtypes using hormone receptor, ki-67 and HER2 expression are commonly used in clinical practice [32]. Basal-like breast cancers are characterized by the absence or low levels of luminal gene expression, absence of HER2 gene cluster expression, and by the expression of genes usually found in basal or myoepithelial cells of the normal breast, such as the basal epithelial cytokeratins (CKs) 5, 14 and 17. Up to $80 \%$ of basal-like cancers are triple negative and approximately $70 \%$ of triple-negative tumors are basallike. In clinical practice, besides the triple-negative definition, the IHC determination of basal CKs (CK5/6, CK14 and/or CK17), Epidermal Growth Factor Receptor (EGFR) and c-kit have been used as surrogates for the basal-like phenotype [33]. Interestingly, TN tumors that are not basal-like exhibit remarkably similar gene expression compared to non-TN tumors [34]. Also, there is a significant phenotypic overlap between TNBC/ basal-like tumors and BRCA1-associated cancers; $80 \%$ of these cancers are basal-like and in spite of the fact that the majority of basal-like tumors do not harbor a BRCA1 mutation, the BRCA1 pathway is commonly deficient in this group of patients [35]. However, there is considerable heterogeneity among triple-negative/ basal-like cancers as some exhibit strong expression of proliferation genes, whereas others express growth factor receptor pathway genes, with basal-like cancers demonstrating the greatest genetic variation [36, 37].

Following the seminal study by Perou et al, similar studies using microarray-based class discovery analysis led to the recognition of additional subtypes of breast cancer. Claudin-low tumors consist of a distinct molecular subtype, characterized by low-toabsent expression of luminal differentiation markers, enrichment for epithelial-to-mesenchymal transition markers, immune response genes and cancer stem cell-like features. Approximately $5-10 \%$ of breast cancers are claudin-low; among claudin-low tumors, up to $70 \%$ are triple negative, and among TNBC, approximately $30 \%$ are claudin-low [38]. Claudin-low tumors have a response rate to chemotherapy that is intermediate between luminal and basal-like tumors and have a better prognosis compared to basal-like ones. Less common molecular subtypes that are frequently clinically triple negative include the interferon-rich or immunomodulatory (IM) subtype, enriched in genes involved in immune processes and associated with a significantly better prognosis as it may represent the medullary breast cancer and the molecular apocrine cancers [androgen receptor-driven (AR) subtype], which offers the promise of targeted treatment against the AR for a small minority of patients with TNBC [39].

In an effort to decipher the heterogeneity of TNBC and to identify potential treatment targets, Lehmann and colleagues performed specific molecular subtyping of TNBC [40]. Using gene expression analyses, the authors described six distinct and reproducible gene expression subtypes of TNBC with differing biology, including two basal-like (BL1 and BL2), an IM, a mesenchymal, a mesenchymal stem-like (MSL) and a luminal AR (LAR) subtype. These subtypes were found to be enriched in distinct gene expression patterns defined by $M$ features, immune system-related genes, DNA damage response genes, and activated AR signaling (Table 1). Furthermore, in the same study, they identified triple negative cell lines that could serve as models for each subtype and employed pharmacological targeting of the respective driver signaling pathways. Using this approach, they were able to identify putative treatment targets according to the specific subtype (Table 1), as a proof-of-concept that analysis of distinct gene expression signatures can be used to guide treatment selection in TNBC.

PAM50 is a clinically applicable gene expressionbased predictor that robustly identifies the main breast cancer intrinsic subtypes by quantitative measurement 
of 50 genes [41]. To determine the relationship between PAM50 intrinsic and TNBC subtypes described by Lehmann et al, Lehmann and Pietenpol examined 374 TNBC samples and concluded that the majority of TNBC subtypes are primarily classified as basal-like by PAM50 (BL1 99\%, BL2 95\%, IM 84\%, M 97\%). MSL TNBCs are classified as basal-like in $50 \%$ of cases with the remainder being normal-like $(28 \%)$, luminal $B$ (14\%), HER2 (5\%) and luminal A (3\%). LAR TNBCs are mainly composed by HER2 (70\%) and Luminal B (14\%) intrinsic subtypes [42].

\section{Molecular biology}

TN tumors exhibit increased genomic instability and a higher mutation burden compared to luminal breast cancers and multiple genetic aberrations and activation of various pathways have been reported in TNBC. It has been shown that the frequencies of copy-number abnormalities and mutations vary markedly between and within tumors, indicating that mutations can arise at multiple stages of tumor progression [37, 43]. TP53 mutations are the more common genetic events, occurring in approximately $80 \%$ of TNBC cases [43]. Basal-like breast cancer is frequently characterized by activation of the PI3K/PTEN/AKT/mTOR pathway as these tumors more commonly have higher Akt activity, whereas PTEN expression is lower compared to HER2(+) tumors [44]. PIK3CA mutations ( $7 \%$ of all breast cancers in one study but up to $34 \%$ in others) or copy number gain (14\% of breast cancers) do not seem to correlate to any specific clinicopathologic characteristics. On the contrary, breast cancers with PTEN loss $(28 \%$ of breast cancers), which is mutually exclusive to PIK3CA mutations and/or Akt activation (observed in $24 \%$ of breast cancers), are more often basal-like. Of note, there seems to be an association between germline BRCA1 mutations and PTEN loss [45]. Interestingly, Shah et al [37] demonstrated that molecular aberrations in TP53, PIK3CA and PTEN, are involved in the early stages of breast-cancer development.

The retinoblastoma protein $(\mathrm{Rb})$ regulates the G1 phase progression of the cell cycle as its hypophosphorylated form binds to and silences the E2F transcription factor, thus blocking passage through the restriction point. Inactivation of $\mathrm{Rb}$ due to low gene expression levels and loss of heterozygosity is found in up to $72 \%$ of basal-like BCs. As apoptosis downstream of $\mathrm{Rb}$ loss is regulated by p53 and inactivation of this tumor suppressor is common in basal-like breast cancer, it has been shown that combined mutation is oncogenic for various breast tumors including TNBC [46].
Other pathways have also been implicated in the biology of TNBC, such as the mitogen-activated protein kinase pathway, with a mutation rate of $5 \%$ for $B R A F, 2 \%$ for KRAS and $2 \%$ for HRAS in one study [47]. Moreover, amplifications of fibroblast growth factor receptor 1 (FGFR1) occur in $9 \%$ of TNBCs and of FGFR2 in 4\% but mutations are rare. Whether FGFR amplification has clinical implications remains to be seen [42].

Basal-like breast cancer is often diagnosed among patients with BRCA1 germline mutations. In addition, up to $20 \%$ of basal-like cancers harbor a BRCA gene mutation, more commonly in BRCA1, compared to only $6 \%$ among all breast cancers [48]. The observed similarities between the two entities go beyond the common expression of basal cell CKs; unlike BRCA2associated tumors and other phenotypes of sporadic breast cancer, BRCA1-associated tumors and TNBC rarely harbor cyclin D1 amplification, express lower levels of p27 and higher levels of S-phase kinaseassociated protein 2, cyclin E, fascin, caveolins 1 and 2, osteonectin, and caspase 3 and harbor a defect in the maintenance of normal chromosome $X$ inactivation [49]. Moreover, sporadic TNBC display clinical and molecular similarities to BRCA1-associated breast cancers and the BRCA1 pathway may be dysfunctional in these tumors despite the absence of somatic mutations, possibly due to epigenetic alterations, a concept termed "BRCAness" [50]. However, this notion remains controversial and of unproven clinical significance.

The LAR subtype seems to be enriched for PIK3CA mutations and shares similar features with ER-/HER2+ breast cancers, such as apocrine morphology and $A R$ expression.

\section{Treatment}

\section{Current approach}

In general, the treatment approach of TNBC, including decisions concerning surgery, radiotherapy, adjuvant or neoadjuvant chemotherapy and the treatment of metastatic disease, is similar to other phenotypes of breast cancer and follow the same principles and guidelines. Two important differences that may lead to increased use of neoadjuvant chemotherapy for this subtype are the more advanced stage at presentation and the increased chemosensitivity of TNBC. Since endocrine manipulation and anti-HER2 agents have no effect on TNBC, chemotherapy remains the main systemic treatment. However, newer targeted treatments for this disease are emerging.

Adjuvant Chemotherapy: According to the National Comprehensive Cancer Network (NCCN) practice 
guidelines, adjuvant chemotherapy is recommended for women with TNBC with node-negative disease and primary tumor size $>0.5 \mathrm{~cm}$ or with node-positive disease and any tumor size [51]. The benefit derived from adjuvant chemotherapy is greater for TNBC compared to ER-positive breast cancer. Thus, in one study, adjuvant chemotherapy resulted in absolute increases of $23 \%$ versus $7 \%$ in disease-free and $17 \%$ versus $4 \%$ in overall survival in patients with TNBC and ER-positive tumors, respectively [52]. Moreover, the addition of taxanes (either docetaxel or paclitaxel) to standard anthracycline-based adjuvant chemotherapy regimens has been shown to offer a higher benefit to TNBC compared to other subtypes in at least three studies [53-55]. However, two questions remain unanswered regarding adjuvant chemotherapy in TNBC: it is unknown whether there is a single regimen that is superior to others and there are no data concerning the optimal management of patients with node-negative TNBC and primary tumor size $<0.5 \mathrm{~cm}$.

Neoadjuvant Chemotherapy: As with adjuvant chemotherapy, most data regarding the use of neoadjuvant chemotherapy in patients with TNBC are derived from retrospective subgroup analyses of trials that include all breast cancer phenotypes, a fact that may lead to underpowered investigation. The disassociation between increased chemosensitivity and dismal outcomes termed "the triple-negative paradox" has been well described $[5,56]$. Several trials have shown increased rates of pathologic complete response ( $p C R$ ) after neoadjuvant chemotherapy for patients with TNBC compared to other breast cancers [25,57]. However, similar to other subtypes, initial chemoresistance to neoadjuvant chemotherapy predicts extremely poor pCR rates despite crossover to non-crossresistant agents [57].

$\mathrm{pCR}$ rates have been consistently shown to closely correlate with survival outcomes in patients with TNBC $[25,58]$. Definitions of pCR vary, but the absence of both invasive and in situ carcinoma after neoadjuvant chemotherapy seems to best predict overall survival. The latter is rather surprising considering that in situ cancer is theoretically treated after surgery [59] and may reflect invasive cancer foci that are undetected during the pathologic examination. Relapse rates for patients with TNBC who do not achieve pCR are up to $50 \%$ at 5 years $[58,59]$. Finally, the increased pCR rates among TNBC raise two important and, as yet, unanswered questions: whether there is a patient subgroup that achieves pCR and could omit completion nodal dissection and/or locoregional radiation and when is the best time to perform sentinel lymph node biopsy, before or after neoadjuvant chemotherapy.
TNBC subtyping may have a predictive value for response to cytotoxic chemotherapy. For example, Masuda and colleagues [60] performed a retrospective analysis of 130 TNBC cases treated with neoadjuvant adriamycin/cyclophosphamide/paclitaxel-containing regimen. The overall $\mathrm{pCR}$ rate was only $28 \%$, but there was considerable difference in the $\mathrm{pCR}$ rate according to subtypes. BL1 had a 52\% pCR rate; BL2 0\%, LAR $10 \%$; and MSL 23\%. In addition, the specific TNBC subtype was an independent predictor of pCR status $(p=0.022)$. Similar findings were reported by another group that subtyped 163 cases considered to be TNBC in The Cancer Genome Atlas [61].

\section{Targeted treatment}

With a better understanding of the biology of TNBC, it is clear that the heterogeneity of this disease precludes one targeted agent to be active in all patients. Categorizing patients into biologically homogenous groups is of paramount importance for the development of more effective targeted agents. Nanostring's PAM50based testing could be of help towards this direction $[37,62]$. As shown in Table 1, multiple putative targeted treatments are currently under evaluation in specific TNBC subtypes; it is reasonable to assume that the heterogeneity of TN tumors should be translated into differential management. Furthermore, the observed multiclonality of TNBC poses a significant challenge, since targeting a single clone with an actionable mutation could probably fail to result in a clinically meaningful response [63,64]. Efforts for targeted treatment approaches in TNBC include the following:

Platinum salts: Consistent with the concept of "BRCAness", platinum agents are hypothesized to be effective in TNBC due to the impaired DNA repair mechanisms of these tumors. However, results have been inconsistent: in the GEICAM/2006-03 trial, the addition of carboplatin to docetaxel after initial neoadjuvant chemotherapy with epirubicin and cyclophosphamide resulted in a statistically nonsignificant increase of pCR (35\% versus $30 \%, P=0.61)$ [65]. In another trial, the use of cisplatin in the adjuvant or neoadjuvant setting resulted in increased clinical complete response rates but data regarding $\mathrm{PCR}$ were not reported [66]. Finally, in the GeparSixto trial, the addition of carboplatin to neoadjuvant chemotherapy with paclitaxel, non-pegylated liposomal doxorubicin and bevacizumab, improved $\mathrm{pCR}$ rates in patients with TNBC [53.2\% versus $36.9 \%(P=0.005)$ ] [67]. The above data show that the use of platinum agents in the neoadjuvant setting of TNBC shows promise; however, prospective randomized Phase III trials are currently lacking. On the other hand, in the metastatic disease, 
Table 1. TNBC subtypes as described in the Cancer Genome Atlas project, their genomic characteristics and putative targeted treatments.

\begin{tabular}{|c|c|c|}
\hline Molecular Subtype & Genetic Characteristics & Putative Targeted Treatments \\
\hline Basal-like 1 & $\begin{array}{l}\text { Cell proliferation pathways, increased cell division and } \\
\text { absent cell-cycle checkpoint }\end{array}$ & $\begin{array}{c}\text { Platinum salts, PARP inhibitors, increased } \\
\text { chemosensitivity }\end{array}$ \\
\hline Basal-like 2 & Growth factor pathways & $\begin{array}{l}\text { Growth factor inhibitors, increased } \\
\text { chemosensitivity }\end{array}$ \\
\hline Immunomodulatory & Immune pathways & Platinum salts, PARP inhibitors \\
\hline Mesenchymal & $\begin{array}{l}\text { Cell motility and differentiation pathways, low } \\
\text { proliferation gene expression }\end{array}$ & $\begin{array}{l}\text { mTOR inhibitors, Src inhibitors, growth factor } \\
\text { inhibitors }\end{array}$ \\
\hline Mesenchymal stem-like & $\begin{array}{l}\text { Increased growth factor signaling and angiogenesis, } \\
\text { low proliferation gene expression }\end{array}$ & PI3K and mTOR inhibitors, growth factor inhibitors \\
\hline Luminal androgen receptor & $\begin{array}{l}\text { Hormonal pathways strongly expressed, regulated by } \\
\text { the androgen receptor }\end{array}$ & AR antagonists, PI3K and mTOR inhibitors \\
\hline
\end{tabular}

cisplatin improved outcomes when added to second line metronomic methotrexate and cyclophosphamide in a single-institution, randomized Phase II trial $(66 \%$ versus $33 \%$ response rate, 13 versus 7 months time to progression, 16 versus 12 months overall survival) [68] PARP inhibitors: Poly-ADP ribose polymerase (PARP) inhibition causes the accumulation of DNA strand breaks in BRCA-deficient tumors and eventually leads to cell death [69]. PARP inhibitors have been tested both in tumors with somatic BRCA mutations and in TNBCs due to the perceived "BRCAness" of these tumors; however, results are inconclusive. In one trial, olaparib resulted in a progression-free survival (PFS) of 5.7 months in patients with $B R C A 1$ or $B R C A 2$ mutation [70]. In another trial, veliparib combined with temozolomide achieved a response rate of only $7 \%$ among patients with TNBC, whereas all responders had germline BRCA mutations [71]. Iniparib, initially considered a PARP inhibitor, failed to improve overall and PFS when added to carboplatin and gemcitabine in patients with TNBC [72]. However, the PARP inhibitory action of iniparib has since been proven false, as it is now thought to act by increasing reactive oxygen species. These early results show that PARP inhibition may have a role in the treatment of the BRCA-mutated subgroup of TNBC [73].

P53: The p53 family of transcription factors ( $p 53$, $p 63, p 73)$, are key regulators of signaling pathways that regulate cell development and tumor suppressive processes [74]. P53 is mutated in $>70 \%$ of TNBCs. In vitro studies have shown that p63 activation leads to increased sensitivity of breast cancer cells, especially TNBC cells to cisplatin, whereas p73 exhibits structural homology to p53. In model cell lines, rapamycin, an mTOR inhibitor, increases p73 occupancy at a subset of its binding sites [74]. Based on these preclinical observations, a randomised Phase II study of neoadjuvant chemotherapy paclitaxel/cisplatin+/everolimus has been initiated (NCT00930930) [76].

PI3K inhibition: Preclinical data have shown that TNBC cells, especially those of the LAR subtype that harbor PI3K mutations more frequently, display strong sensitivity to PI3K pathway inhibitors [77]. Combinations of $\mathrm{PI} 3 \mathrm{~K}$ inhibitors and cisplatin show synergistic decrease in the viability of TNBC cells with significant decreases in pAKT and pS6 levels and elevation in cleaved PARP. Moreover, PI3K inhibitors downregulate BRCA1/2 expression, thus sensitizing BRCA-proficient tumors to PARP inhibition [78]. A Phase I study of a pan-PI3K with a PARP inhibitor combination is currently ongoing in metastatic TNBC.

MEK inhibition: Activation of the RAS/MEK pathway seems to play an important role in basal-like cells survival [79]. Preclinical data suggest that TNBCs with PTEN expression and low activation of DUSP4 (a negative regulator of ERK1 and ERK2 kinases) have a dependency on RAS-ERK activation [80] and could be sensitive to combinations of chemotherapy with MEK inhibitors.

Angiogenesis inhibitors: Vascular Endothelial Growth Factor (VEGF) is the most important mediator of tumor angiogenesis and expression of VEGF has been found to be higher in TNBC compared to nonTNBC [81]. In the neoadjuvant setting, a prespecified subgroup analysis of the GeparQuinto trial showed improved $\mathrm{pCR}$ rates with the addition of bevacizumab, an anti-VEGF monoclonal antibody, to the standard neoadjuvant chemotherapy in TNBC patients $(39.3 \%$ versus $27.9 \% \quad P=0.003$ ) [82]. Another large Phase III trial, NSABP-B40, showed the opposite results in the same setting ( $\mathrm{pCR} 51.3 \%$ versus $47.3 \%$ with and without bevacizumab, respectively, $P=0.44$ ) [83]. In metastatic disease, subgroup analyses from the AVADO and E2100 
trials showed that bevacizumab added to standard chemotherapy doubled PFS in patients with TNBC, whereas the RIBBON-1 trial showed no difference [84]. A meta-analysis of these three first-line trials showed an improvement in PFS by 2.7 months with the addition of bevacizumab $(P<0.0001)$ [85]. Finally, in the second-line setting, bevacizumab added to chemotherapy (taxane, gemcitabine, capecitabine or vinorelbine) significantly improved PFS (6 versus 2.7 months, $P=0.0006$ ) and the difference in overall survival bordered but did not reach clinical significance (17.9 versus 12.6 months, $P=0.0534$ ) [86].

Tyrosine kinase inhibitors targeting the VEGF receptor have also been tested in TNBC in phase II trials. Sunitinib and sorafenib as single agents showed limited activity and significant toxicity when combined with chemotherapy [87-90]. However, in at least one trial, the combination of sorafenib with capecitabine improved PFS over capecitabine alone (4.3 versus 2.5 months).

EGFR inhibitors: Since TNBC frequently stains immunohistochemically positive for EGFR, various agents targeting the EGFR pathway have been tested in this subtype. Cetuximab, a monoclonal antibody that targets the extracellular domain of EGFR has been tested in three Phase II trials that enrolled metastatic TNBC patients in various combinations, mainly with platinum salts (cisplatin, carboplatin, irinotecan and carboplatin, respectively). However, activity was modest with improvements in response rates but no improvement in overall survival and PFS in the first two trials [91-93]. Lapatinib, an EGFR and HER2 inhibitor, failed to improve outcomes when combined with paclitaxel in metastatic patients [94]. Erlotinib, an EGFR inhibitor, showed promising activity when combined with docetaxel and carboplatin in the neoadjuvant setting [95].

Src inhibitors: Expression profiling suggests that basal-like cancers may be preferentially sensitive to src inhibition. In a Phase II trial, dasatinib, a src inhibitor that also potently inhibits BCR-ABL, showed a clinical benefit rate of $9.2 \%$ among patients with TNBC prior exposure to anthracycline and taxane therapy [96].

mTOR inhibitors: As previously stated, the PI3K/ $\mathrm{PTEN} / \mathrm{AKT} / \mathrm{mTOR}$ pathway is frequently activated in TNBC. Everolimus, an mTOR inhibitor approved for use in ER-positive breast cancer, kidney cancer and neuroendocrine tumors, did not improve outcomes in the neoadjuvant setting when combined with standard chemotherapy [97]. In the metastatic setting, 25 pretreated patients (including platinum pretreated) received a combination of carboplatin and everolimus; disease control rate was $36 \%$ (including one complete and six partial responses) and median PFS was 3 months [98].

Antiandrogens: In one recent Phase II study, only AR-positive TNBCs were treated with the AR antagonist bicalutamide and a $19 \%$ clinical benefit rate was observed, leading to an ongoing trial testing the more potent AR antagonist enzalutamide [99].

\section{Future prospects and conclusion}

Despite improvements in the understanding of the biology of triple-negative breast cancer, the prognosis is poor and novel treatment strategies remain an unmet need. Despite increased chemosensitivity, a proportion of patients with early TNBC do not derive benefit from adjuvant or neoadjuvant chemotherapy. The recognition of $\mathrm{pCR}$ as an appropriate surrogate for measuring longterm outcomes facilitates the optimization of treatment in early TNBC. However, the most appropriate treatment for patients with very early tumors (i.e. $<0.5 \mathrm{~cm}$ ) and the optimal sentinel lymph node biopsy strategies are currently unknown. Moreover, unanswered questions remain concerning the role of platinum compounds in the metastatic setting that is being addressed in ongoing trials, such as the Triple Negative Breast Cancer Trial (TNT study, NCT00532727), evaluating the activity of carboplatin versus docetaxel after progression with an anthracycline.

Regarding targeted treatments, it is clear that this approach, thus far, has looked less promising compared to ER-positive and HER2-positive breast cancers. Retrospective evaluations of anti-VEGF treatment demonstrated encouraging improvements in PFS, whereas EGFR, mTOR and src inhibition had minimal activity. However, PI3K and Rb pathway targeting has a strong biologic rationale and ongoing research may yield clinically relevant results. Moreover, PARP inhibition seems to be active mainly in the subset of TNBC that harbor BRCA mutations.

The challenges for the future are to better understand the heterogeneity of TNBC, to appropriately select well-defined patient populations for specific targeted treatments, to discover biomarkers that are predictors of response and to conduct prospective Phase III trials in TNBC-only populations to better evaluate the role of existing targeted treatments. BEATRICE Study [A Study of Avastin (Bevacizumab) Adjuvant Therapy in Triple Negative Breast Cancer (NCT00528567)] and the RESILIENCE study [Phase III Trial Comparing Capecitabine in Combination With Sorafenib or Placebo in the Treatment of Locally Advanced or Metastatic HER2-Negative Breast Cancer (NCT01234337)] are 
evaluating the role of these agents in the adjuvant and metastatic setting of TNBC, respectively.

In conclusion, triple-negative breast cancer exhibits remarkable heterogeneity. The understanding of the distinct molecular subtypes and the signaling pathways underlying its biology may lead to improved outcomes for this poor prognostic subgroup of breast cancer patients through the conduct of rationally designed clinical trials.

The authors report no financial interests or potential conflicts of interest.

\section{References}

[1] Siegel R, Naishadham D, Jemal A. Cancer statistics, 2012. CA Cancer J Clin 2012;62:10-29.

[2] Hammond ME, Hayes DF, Dowsett $M$ et al. American Society of Clinical Oncology/College Of American Pathologists guideline recommendations for immunohistochemical testing of estrogen and progesterone receptors in breast cancer. J Clin Oncol 2010;28:2784-95.

[3] Wolff AC, Hammond ME, Hicks DG et al. Recommendations for human epidermal growth factor receptor 2 testing in breast cancer: American Society of Clinical Oncology/College of American Pathologists clinical practice guideline update. J Clin Oncol 2013;31:3997-4013.

[4] Brenton JD, Carey LA, Ahmed AA et al. Molecular classification and molecular forecasting of breast cancer: ready for clinical application? J Clin Oncol 2005;23:7350-60.

[5] Foulkes WD, Smith IE, Reis-Filho JS. Triple-negative breast cancer. N Engl J Med 2010;363:1938-48.

[6] BoyleP.Triple-negativebreastcancer:epidemiological considerations and recommendations. Ann Oncol 2012;23 Suppl 6:vi7-12.

[7] Trivers KF, Lund MJ, Porter PLetal. The epidemiology of triple-negative breast cancer, including race. Cancer Causes Control 2009;20:1071-82.

[8] Kwan ML, Kushi LH, Weltzien E et al. Epidemiology of breast cancer subtypes in two prospective cohort studies of breast cancer survivors. Breast Cancer Res 2009;11:R31.

[9] Stead LA, Lash TL, Sobieraj JE et al. Triplenegative breast cancers are increased in black women regardless of age or body mass index. Breast Cancer Res 2009; 11:R18.

[10] Carey LA, Perou CM, Livasy CA et al. Race, breast cancer subtypes, and survival in the Carolina Breast Cancer Study. JAMA 2006;295:2492-502.

[11] Huo D, Ikpatt F, Khramtsov A et al. Population differences in breast cancer: survey in indigenous

\section{Author Contributions}

GL: Literature review, drafting of the manuscript, critical revisions

AM: Literature review, drafting of the manuscript

SA: Drafting of the manuscript, critical revisions

African women reveals over-representation of triple-negative breast cancer. J Clin Oncol 2009 Sep;27:4515-21.

[12] Millikan RC, Newman B, Tse CK et al. Epidemiology of basal-like breast cancer. Breast Cancer Res Treat 2008;109:123-39.

[13] Phipps Al, Chlebowski RT, Prentice R et al. Reproductive history and oral contraceptive use in relation to risk of triple-negative breast cancer. J Natl Cancer Inst 2011;103:470-477.

[14] Dolle JM, Daling JR, White E et al. Risk factors for triple-negative breast cancer in women under age 45. Cancer Epidemiol Biomarkers Prev 2009;18:1157-1166.

[15] Pierobon M, Frankenfeld CL. Obesity as a risk factor for triple-negative breast cancers: a systematic review and meta-analysis. Breast Cancer Res Treat 2013;137:307-14.

[16] Chen WY, Rosner B, Hankinson SE et al. Moderate alcohol consumption during adult life, drinking patterns, and breast cancer risk. JAMA 2011;306:1884-90.

[17] Liu Y, Colditz GA, Rosner B et al. Alcohol intake between menarche and first pregnancy: a prospective study of breast cancer risk. J Natl Cancer Inst 2013;105:1571-8.

[18] Cui Y, Miller AB, Rohan TE. Cigarette smoking and breast cancer risk: update of a prospective cohort study. Breast Cancer Res Treat 2006;100:293-9.

[19] Johnson KC, Miller AB, Collishaw NE et al. Active smoking and secondhand smoke increase breast cancer risk: the report of the Canadian Expert Panel on Tobacco Smoke and Breast Cancer Risk (2009). Tob Control 2011;20:e2.

[20] Kabat GC, Kim M, Phipps Al et al. Smoking and alcohol consumption in relation to risk of triple-negative breast cancer in a cohort of postmenopausal women. Cancer Causes Control 2011;22:775-783. 
[21] Livasy CA, Karaca G, Nanda R et al. Phenotypic evaluation of the basal-like subtype of invasive breast carcinoma. Mod Pathol 2006;19:264-71.

[22] Turner NC, Reis-Filho JS. Tackling the diversity of triple-negative breast cancer. Clin Cancer Res 2013;19:6380-8.

[23] Dent R, Trudeau M, Pritchard KI et al. Triple-negative breast cancer: clinical features and patterns of recurrence. Clin Cancer Res 2007;13:4429-34.

[24] Collett K, Stefansson IM, Eide J et al. A basal epithelial phenotype is more frequent in interval breast cancers compared with screen detected tumors. Cancer Epidemiol Biomarkers Prev 2005;14:1108-12.

[25] Edge SB, Byrd DR, Compton CC et al (Eds). AJCC (American Joint Committee on Cancer). Cancer Staging Manual, 7th edition, Springer-Verlag, New York 2010. p.347

[26] Liedtke C, Mazouni C, Hess KR et al. Response to neoadjuvant therapy and longterm survival in patients with triple-negative breast cancer. J Clin Oncol 2008;26:1275-81.

[27] Dent R, Hanna WM, Trudeau M et al. Pattern of metastatic spread in triple-negative breast cancer. Breast Cancer Res Treat 2009;115:423-8.

[28] Lin NU, Vanderplas A, Hughes ME et al. Clinicopathologic features, patterns of recurrence, and survival among women with triple-negative breast cancer in the National Comprehensive Cancer Network. Cancer 2012;118:5463-72.

[29] Hamm C, El-Masri M, Poliquin G et al. A singlecentre chart review exploring the adjusted association between breast cancer phenotype and prognosis. Curr Oncol 2011;18:191-6.

[30] Iwase H, Kurebayashi J, Tsuda $H$ et al. Clinicopathological analyses of triple negative breast cancer using surveillance data from the Registration Committee of the Japanese Breast Cancer Society. Breast Cancer 2010;17:118-24.

[31] Perou CM, Sørlie T, Eisen MB et al. Molecular portraits of human breast tumours. Nature 2000;406:747-52.

[32] Goldhirsch A, Winer EP, Coates AS et al. Personalizing the treatment of women with early breast cancer: highlights of the St Gallen International Expert Consensus on the Primary Therapy of Early Breast Cancer 2013. Ann Oncol 2013;24:2206-23.

[33] Nielsen TO, Hsu FD, Jensen $K$ et al. Immunohistochemical and clinical characterization of the basal-like subtype of invasive breast carcinoma. Clin Cancer Res 2004;10:5367-74.

[34] Prat A, Adamo B, Cheang MC et al. Molecular characterization of basal-like and non-basallike triple-negative breast cancer. Oncologist 2013;18:123-33.

[35] Turner N, Lambros MB, Horlings HM et al. Integrative molecular profiling of triple negative breast cancers identifies amplicon drivers and potential therapeutic targets. Oncogene 2010;29:2013-23.

[36] Rakha EA, Reis-Filho JS, Ellis IO. Basal-like breast cancer: a critical review. J Clin Oncol 2008;26:256881.

[37] Shah SP, Roth A, Goya R et al. The clonal and mutational evolution spectrum of primary triplenegative breast cancers. Nature 2012;486:395-9.

[38] Prat A, Parker JS, Karginova O et al. Phenotypic and molecular characterization of the claudin-low intrinsic subtype of breast cancer. Breast Cancer Res 2010;12:R68.

[39] Sotiriou C, Pusztai L. Gene-expression signatures in breast cancer. N Engl J Med 2009;360:790-800.

[40] Lehmann BD, Bauer JA, Chen X et al. Identification of human triple-negative breast cancer subtypes and preclinical models for selection of targeted therapies. J Clin Invest 2011;121:2750-67.

[41] Parker JS, Mullins M, Cheang MC et al. Supervised risk predictor of breast cancer based on intrinsic subtypes. J Clin Oncol. 2009;27:1160-7.

[42] Lehmann BD, Pietenpol JA. Identification and use of biomarkers in treatment strategies for triple-negative breast cancer subtypes. J Pathol 2014;232:142-50.

[43] Cancer Genome Atlas Network. Comprehensive molecular portraits of human breast tumours. Nature 2012;490:61-70.

[44] Marty B, Maire V, Gravier E et al. Frequent PTEN genomic alterations and activated phosphatidylinositol 3-kinase pathway in basallike breast cancer cells. Breast Cancer Res 2008;10:R101.

[45] López-Knowles E, O’Toole SA, McNeil CM etal. PI3K pathway activation in breast cancer is associated with the basal-like phenotype and cancer-specific mortality. Int J Cancer 2010;126:1121-31.

[46] Jiang Z, Jones R, Liu JC et al. RB1 and p53 at the crossroad of EMT and triple-negative breast cancer. Cell Cycle 2011;10:1563-70.

[47] Hu X, Stern HM, Ge L et al. Genetic alterations and oncogenic pathways associated with breast cancer subtypes. Mol Cancer Res 2009;7:511-22.

[48] Gonzalez-Angulo AM, Timms KM, Liu S et al. Incidence and outcome of BRCA mutations in unselected patients with triple receptor-negative breast cancer. Clin Cancer Res 2011;17:1082-9.

[49] Turner NC, Reis-Filho JS, Russell AM et al. BRCA1 
dysfunction in sporadic basallike breast cancer. Oncogene 2007;26:2126-32.

[50] Turner N, Tutt A, Ashworth A. Hallmarks of 'BRCAness' in sporadic cancers. Nat Rev Cancer 2004;4:814-9.

[51] NCCN Clinical Practice Guidelines in Oncology: Breast Cancer. Version 3.2014. http://www.nccn. org/professionals/physician_gls/pdf/breast.pdf (Accessed on May 4, 2014)

[52] Berry DA, Cirrincione C, Henderson IC et al. Estrogen-receptor status and outcomes of modern chemotherapy for patients with node-positive breast cancer. JAMA 2006;295:1658-67.

[53] Hayes DF, Thor AD, Dressler LG et al. HER2 and response to paclitaxel in nodepositive breast cancer. N Engl J Med 2007;357:1496-506.

[54] Ellis P, Barrett-Lee P, Johnson L, et al. Sequential docetaxel as adjuvant chemotherapy for early breast cancer (TACT): an open-label, phase III, randomised controlled trial. Lancet 2009;373:168192.

[55] Martín M, Rodríguez-Lescure A, Ruiz A et al. Molecular predictors of efficacy of adjuvant weekly paclitaxel in early breast cancer. Breast Cancer Res Treat 2010;123:149-57.

[56] Carey LA, Dees EC, Sawyer L et al. The triple negative paradox: primary tumor chemosensitivity of breast cancer subtypes. Clin Cancer Res 2007;13:2329-34.

[57] Huober J, von Minckwitz G, Denkert C et al. Effect of neoadjuvant anthracycline-taxane-based chemotherapy in different biological breast cancer phenotypes: overall results from the GeparTrio study. Breast Cancer Res Treat 2010;124:133-40.

[58] Guarneri V, Broglio K, Kau SW et al. Prognostic value of pathologic complete response after primary chemotherapy in relation to hormone receptor status and other factors. J Clin Oncol 2006;24:1037-44.

[59] von Minckwitz G, Untch M, Blohmer JU et al. Definition and impact of pathologic complete response on prognosis after neoadjuvant chemotherapy in various intrinsic breast cancer subtypes. J Clin Oncol 2012;30:1796-804.

[60] Masuda H, Baggerly KA, Wang Y et al. Differential response to neoadjuvant chemotherapy among seven triple-negative breast cancer molecular subtypes. Clin Cancer Res 2013;19:5533 - 5540.

[61] Chen X, Li J, Gray WH et al. TNBCtype: a subtyping tool for triple-negative breast cancer. Cancer Inform 2012;11:147-56.

[62] Weigelt B, Mackay A, A'Hern R et al. Breast cancer molecular profiling with single sample predictors: a retrospective analysis. Lancet Oncol 2010;11:33949.

[63] Ding L, Ellis MJ, Li S et al. Genome remodelling in a basal-like breast cancer metastasis and xenograft. Nature 2010;464:999-1005.

[64] Nik-Zainal S, Van Loo P, Wedge DC et al. The life history of 21 breast cancers. Cell 2012;149: 9941007.

[65] Silver DP, Richardson AL, Eklund AC et al. Efficacy of neoadjuvant cisplatin in triple-negative breast cancer. J Clin Oncol 2010;28:1145-53.

[66] Sirohi B, Arnedos M, Popat S et al. Platinum-based chemotherapy in triple-negative breast cancer. Ann Oncol 2008;19:1847-52.

[67] von Minckwitz G, Schneeweiss A, Loibl S et al. Neoadjuvant carboplatin in patients with triplenegative and HER2-positive early breast cancer (GeparSixto; GBG 66): a randomised phase 2 trial. Lancet Oncol 2014; Published Online May 1.

[68] Bhattacharyya GS, Basu S, Agarwal V et al. Single institute phase II study of weekly cisplatinum and metronomic dosing of cyclophosphamide and methotrexate in second line metastatic breast cancer triple-negative. Eur $\mathrm{J}$ Cancer 2009;7:18:Abstr 41LBA

[69] Tentori L, Graziani G. Chemopotentiation by PARP inhibitors in cancer therapy. Pharmacol Res 2005;52:25-33.

[70] Tutt A, Robson M, Garber JE et al. Oral poly(ADPribose) polymerase inhibitor olaparib in patients with BRCA1 or BRCA2 mutations and advanced breast cancer: a proof-of-concept trial. Lancet 2010;376:235-44.

[71] Isakoff SJ, Overmoyer B, Tung NM, et al A phase II trial of the PARP inhibitor veliparib (ABT888) and temozolomide for metastatic breast cancer. J Clin Oncol 2010;28(15s):abstr 1019.

[72] O'Shaughnessy J, Schwartzberg LS, Danso MA et al. A randomized phase III study of iniparib (BSI201) in combination with gemcitabine/carboplatin $(\mathrm{G} / \mathrm{C})$ in metastatic triple-negative breast cancer (TNBC). J Clin Oncol 2011;29(81s):abstr 1007

[73] Gelmon K, Dent R, Mackey JR et al. Targeting triplenegative breast cancer: optimizing therapeutic outcomes. Ann Oncol 2012;23:2223-34.

[74] Barbieri CE, Pietenpol JA. p63 and epithelial biology. Exp Cell Res 2006;312:695-706.

[75] Rosenbluth JM, Mays DJ, Jiang A et al. Differential regulation of the $p 73$ cistrome by mammalian target of rapamycin reveals transcriptional programs of mesenchymal differentiation and tumorigenesis. Proc Natl Acad Sci USA 2011;108:2076-81.

[76] Mayer IA, Jovanovic B, Abramson VG et al. A 
randomized phase II neoadjuvant study of cisplatin, pac- litaxel with or without everolimus (an mTOR inhibitor) in patients with stage II/III triple-negative breast cancer (TNBC). San Antonio Breast Cancer Symposium 2013; Poster Discussion PD1-6.

[77] Gonzalez-Angulo AM, Stemke-Hale K, Palla SL et al. Androgen receptor levels and association with PIK3CA mutations and prognosis in breast cancer. Clin Cancer Res 2009;15:2472-8.

[78] Ibrahim YH, Garcia-Garcia C, Serra V et al. PI3K inhibition impairs BRCA1/2 expression and sensitizes BRCA-proficient triple-negative breast cancer to PARP inhibition. Cancer Discov 2012;2:1036-47.

[79] Hoeflich KP, O'Brien C, Boyd Z et al. In vivo antitumor activity of MEK and phosphatidylinositol 3-kinase inhibitors in basal-like breast cancer models. Clin Cancer Res 2009;15:4649-64.

[80] Balko JM, Cook RS, Vaught DB et al. Profiling of residual breast cancers after neoadjuvant chemotherapy identifies DUSP4 deficiency as a mechanism of drug resistance. Nat Med 2012;18:1052-9.

[81] Linderholm BK, Hellborg $H$, Johansson $U$ et al. Significantly higher levels of vascular endothelial growth factor (VEGF) and shorter survival times for patients with primary operable triple-negative breast cancer. Ann Oncol 2009;20:1639-1646.

[82] von Minckwitz G, Eidtmann H, Rezai M et al. Neoadjuvant chemotherapy and bevacizumab for HER2-negative breast cancer. N Engl J Med 2012; 366:299-309.

[83] Bear HD, Tang G, Rastogi P et al. The effect on pCR of bevacizumab and/or antimetabolites added to standard neoadjuvant chemotherapy: NSABP protocol B-40. J Clin Oncol 2011;29:Abstr LBA 1005.

[84] O'Shaughnessy J, Dieras V, Glaspy J et al. Comparison of subgroup analyses of PFS from three phase III studies of bevacizumab in combination with chemotherapy in patients with HER2-negative metastatic breast cancer (MBC). Cancer Res 2009;69:Abstr 207.

[85] O'Shaughnessy J, Romieu G, Dieras V et al. Metaanalysis of patients with triple-negative breast cancer (TNBC) from three randomized trials of first-line bevacizumab (BV) and chemotherapy treatment for metastatic breast cancer (MBC). Cancer Res 2010;70:Abstr P6-12-03.

[86] Brufsky A, Valero V, Tiangco B et al. Impact of bevacizumab (BEV) on efficacy of second-line chemotherapy (CT) for triple-negative breast cancer (TNBC): analysis of RIBBON-2. J Clin Oncol 2011;29:Abstr 1010.
[87] Burstein HJ, Elias AD, Rugo HS et al. Phase II study of sunitinib malate, an oral multitargeted tyrosine kinase inhibitor, in patients with metastatic breast cancer previously treated with an anthracycline and a taxane. J Clin Oncol 2008;26:1810-1816

[88] Moreno-Aspitia A, Morton RF, Hillman DW et al. Phase II trial of sorafenib in patients with metastatic breast cancer previously exposed to anthracyclines or taxanes: North Central Cancer Treatment Group and Mayo Clinic Trial N0336. J Clin Oncol 2009;27:11-15.

[89] Crown J, Dieras V, Staroslawska E et al. Phase III trial of sunitinib (SU) in combination with capecitabine $(C)$ versus $C$ in previously treated advanced breast cancer (ABC). J Clin Oncol. 2013;31:2870-8.

[90] Baselga J, Roche H, Costa F et al. SOLTI-0701: a multinational double-blind, randomized phase $2 \mathrm{~b}$ study evaluating the efficacy and safety of sorafenib compared to placebo when administered in combination with capecitabine in patients with locally advanced or metastatic breast cancer (BC). Cancer Res 2009;69:Abstr 45

[91] Baselga J, Stemmer S, Pego A et al. Cetuximab + cisplatin in estrogen receptor-negative, progesterone receptor-negative, HER2-negative (triplenegative) metastatic breast cancer: results of the randomized phase II BALI-1 trial. Cancer Res 2010;70:Abstr PD01-01.

[92] O'Shaughnessy J, Weckstein D, Vukelja S et al. Preliminary results of a randomized phase II study of weekly irinotecan/carboplatin with or without cetuximab in patients with metastatic breast cancer. Breast Cancer Res Treat 2007;106:S32:Abstr 308.

[93] Carey LA, Rugo HS, Marcom PK et al. TBCRC 001: EGFR inhibition with cetuximab added to carboplatin in metastatic triple-negative (basal-like) breast cancer. J Clin Oncol 2008;26:Abstr 1009.

[94] Finn RS, Press MF, Dering $J$ et al. Estrogen receptor, progesterone receptor, human epidermal growth factor receptor 2 (HER2), and epidermal growth factor receptor expression and benefit from lapatinib in a randomized trial of paclitaxel with lapatinib or placebo as first-line treatment in HER2negative or unknown metastatic breast cancer. J Clin Oncol 2009;27:3908-3915.

[95] Sharma P, Khan QJ, Kimler BF et al. Results of a phase II study of neoadjuvant platinum/taxane based chemotherapy and erlotinib for triple negative breast cancer. Cancer Res 2010;70:Abstr P1-11-07.

[96] Finn R, Bengala C, Ibrahim N, et al. Phase II trial of 
dasatinib in triple-negative breast cancer: results of study CA180059. Cancer Res 2009; 69:Abstr 3118.

[97] Gonzalez-Angulo AM, Green MC, Murray JL et al. Open label, randomized clinical trial of standard neoadjuvant chemotherapy with paclitaxel followed by FEC (T-FEC) versus the combination of paclitaxel and RAD001 followed by FEC (TR-FEC) in women with triple receptor-negative breast cancer (TNBC). J Clin Oncol 2011;29:Abstr 1016.

[98] Singh JC, Novik Y, Stein S et al. Phase 2 trial of everolimus and carboplatin combination in patients with triple negative metastatic breast cancer. Breast Cancer Res 2014;16:R32.

[99] Gucalp A, Tolaney SM, Isakoff SJ et al. Targeting the androgen receptor (AR) in women with $A R+$ ER-/PR- metastatic breast cancer (MBC). J Clin Oncol 2012;30 suppl:abstr 1006. 